\title{
Dynamic of interactive model for information propagation across social networks media
}

\author{
Yaming Zhang ${ }^{1,3}$, Fei Liu ${ }^{1,2^{*}}$, Yaya H. Koura ${ }^{*}$ (D) and Hao Wang
}

\section{"Correspondence:}

liufei937087578@126.com:

2689395039@qq.com

'School of Economics and

Management, Yanshan University, Qinhuangdao, China

${ }^{2}$ School of Public Administration, Hengshui University, Hengshui,

China

Full list of author information is available at the end of the article

\begin{abstract}
Controlling information diffusion or propagation through social networks can be challenging when dealing with information related to a subject of highest interest for the public. The complexity level of control depends on subject importance, users' dynamic, and network structure. When two published messages or pieces of information share the same interest for targeted readers, analyzing their propagation dynamic for control and prediction is of great interest. This article proposes to model, based on a modified interactive system with Holling type functional response, the dynamic of underlying relationship between two broadcasted messages traveling through social networks media. We showed in the qualitative analysis of the proposed model that system could be stable at certain conditions, and the model-system exhibited very rich dynamical behavior. Numerical simulation results validated theoretical analyses and suggested adapting resources harvesting and assimilation efficiency for an authoritative message to stabilize the system and control the dissemination of information in a closed environment.
\end{abstract}

Keywords: Information propagation; Social network; Competitive interaction; Logistic model; Holling type II; Stability analysis

\section{Introduction}

Avoiding overdiffusion or propagation of broadcasted messages or information in social networks media or platform can be necessary and needed in preventing disinformation, panic, or overreaction due to content sensibility in a closed environment. On most of social media platforms, information propagation may follow a random pattern relying on the size of the network, users' dynamic, and publishers' ties or importance [1-4]. It has become challenging to predict and control the speed of diffusion and dissemination of information in larger scale and complex networks such as today's social networks. The omnipresence of connectivity and wide spread of intelligent devices are among multiple reasons that may explain this complexity. As investigated by numerous authors, most of the studies related to information propagation, rumors diffusion, and so forth have been focusing on information speed of diffusion, readership growth rate, and network topology. Some researchers investigated node properties to analyze the influence each node has on

(c) The Author(s) 2020. This article is licensed under a Creative Commons Attribution 4.0 International License, which permits use sharing, adaptation, distribution and reproduction in any medium or format, as long as you give appropriate credit to the original author(s) and the source, provide a link to the Creative Commons licence, and indicate if changes were made. The images or other third party material in this article are included in the article's Creative Commons licence, unless indicated otherwise in a credit line to the material. If material is not included in the article's Creative Commons licence and your intended use is not permitted by statutory regulation or exceeds the permitted use, you will need to obtain permission directly from the copyright holder. To view a copy of this licence, visit http://creativecommons.org/licenses/by/4.0/. 
information dissemination in a period of crisis for example, relying on epidemic control models and social network analysis techniques and methods [5-9].

We are interested in this article in analyzing the influence different pieces of information may have on each other when they are related to the same topic and have the same interest for the audience or readership. Identifying the type and nature of underlying relationship between interacting entities is a key in controlling and predicting system behavior as stipulated in many published articles in related literature [10-15].

In this article, we propose to consider the impact of social network users' behavior on information propagation dynamic when two influential publishers publish two messages or pieces of information on a social media platform. We consider the published messages to be related to the same topic, thus sharing relatively the same interest for readers or followers. The published messages may reach a larger audience, depending on publishers' ties and importance in respect to the number of followers. Readers may follow both publishers; consequently, one published message related to the same subject may drive readers to do more comparisons for enrichment of viewpoint and knowledge about the respective subject. As a result, one published message may lead to discovery and contact with more messages related to the same topic from different publishers. Depending on how reliable readers consider respective publishers, they will repost, transmit, or recommend the piece of information to their own followers [16-20]. This phenomenon can be assimilated to a predator-prey type relationship in which messages or information that increase respective readership per time unit could be considered as predator. As in the classical dynamic social network analysis, users or nodes have random behavior and may move to different locations, virtual groups, etc. We consider the publishers to share audience and the readers to join randomly any publishers' open group.

Traditional logistic Lotka-Volterra based interactive systems are commonly used to model interactions occurring in a system where entities share available resources. The advantage of such systems is that they exhibit rich dynamical behavior and have many applications in the real world, such as economic ties, interconnected markets, applied engineering sciences, ecology, etc. Models with Holling type functional responses are well spread and there are large varieties of modified models adjusted to formalize specific ties or relationship in dynamical systems. Depending on studied systems and underlying interaction types, the outcome of interaction may be simple or complex. Holling type functional response commonly allows better control of resources accessibility, harvesting time, assimilation efficiency, and so forth [21-24].

Controlling rumor and fake news overdiffusion through social media is a recurrent problem that needs to be addressed from different perspectives for control purposes. Measuring and reducing information speed of diffusion in nowadays society have become challenging due to social network popularity and widespread Internet network. The upcoming of next generation mobile and intelligent devices along with the Internet of things may even bring more challenges from management and control standpoint. This investigation proposes an insight into network resources administration and information diffusion. Whether we deal with real news or rumors, this article provides a new angle of investigation to describe and solve existing issues and challenges from resources optimization and control perspectives. Results of this work could be used to determine the effect authoritative information could have on related persistent propagating rumors traveling through social networks media, such as Weibo, WeChat, etc. Particularly, in a period of 
crisis where a decision about the content related to national security or social wellbeing has to be made to establish or restore the truth. The main motivation of this investigation is to provide supporting evidence on the importance of controlling overdiffusion of sensible and potentially dangerous contents across social media platforms and promoting the truth.

By performing qualitative analysis of the proposed competitive model, we found that, under certain conditions, the system could be stable and both published pieces of information could coexist peacefully. Furthermore, the dynamic of this particular competition in a closed environment is impacted directly by readers' behavior, content nature, the importance of related subject in respect to readers' interest, and network state in terms of resources availability as shown in numerical simulation results. Adjusting diffusion efficiency or publishing higher interest information through certain nodes may boost given message propagation efficiency and improve the publisher's esteem or reliability.

\section{Model}

Let $M_{1}$ and $M_{2}$ be the number of views of respectively the first and second message at time $t$. If we consider there is interconnection between readers, then people who read $M_{1}$ first may or may not look at $M_{2}$ content for crosschecking purpose for example and viceversa. This behavior depends on content attractiveness or interest for the reader. Considering there is an upper limit in the number of views for each posted message, we formalize this limitation using logistic terms in both $M_{1}$ and $M_{2}$. Each message will increase respective views or population size, using ecology terms, if the number of views increases per time unit, the rate of increase being proportional to the per capita total number of views at time $t$. A model system is given by

$$
\begin{aligned}
& \frac{d M_{1}}{d t}=M_{1}\left[a_{1}\left(V-n M_{1}\right)\left(1-\frac{M_{1}}{V}\right)-\frac{a_{2} M_{2}}{1+a_{3} M_{1}}\right], \\
& \frac{d M_{2}}{d t}=M_{2}\left[b_{1}\left(1-\frac{m M_{2}}{V}\right)+\frac{b_{2} M_{1}}{1+a_{3} M_{1}}\right]
\end{aligned}
$$

where $a_{1}, a_{2}, a_{3}, b_{1}, b_{2}, n, m$, and $V$ are positive constant parameters, $a_{1}$ and $b_{1}$ represent respectively the natural growth coefficients of $M_{1}$ and $M_{2} . a_{2}$ corresponds to the interaction effect of $M_{2}$ on $M_{1}$, and $b_{2}$ is positive feedback resulting from this interaction. $a_{3}$ formalizes assimilation efficiency and harvesting effort in respect to the time needed to capture and consume resources. $n$ is a control parameter modeling the threshold when $M_{1}$ reaches the critical number of views. $m$ is a control parameter modeling the effect of promotion from which $M_{2}$ benefits. $V$ is the maximum number of views each message can reach. This factor is incorporated in the proposed model for practical and analysis purposes, in respect to a social network's finite number of views.

It is clear that:

(i) Both $M_{1}$ and $M_{2}$ increase density or number of views as far as $0<M_{1}(t)<V<V / n$, and $0<M_{2}(t)<V<V / m$ as far as $0<m, n<1$.

(ii) $a_{2} M_{2} /\left(1+a_{3} M_{1}\right)$ value depends on $a_{2}$ and $a_{3}$ for a larger $M_{1}$ population or the number of views. $M_{2}$ will thrive only if interaction is intensive, meaning when there is a significant proportion of readers who only read one message and never double check, trusting respective publisher. On the other hand, $M_{1}$ will suffer less from predation if $M_{2}$ is weaker or has relatively less views. 
Set

$$
\begin{aligned}
& \frac{d M_{1}}{d t}=f_{1}\left(M_{1}, M_{2}\right), \\
& \frac{d M_{2}}{d t}=f_{2}\left(M_{1}, M_{2}\right),
\end{aligned}
$$

such that $f_{1}\left(M_{1}, M_{2}\right)$ and $f_{2}\left(M_{1}, M_{2}\right)$ are continuously derivable functions for $M_{1}, M_{2}>0$.

Then we can write

$$
\begin{aligned}
\frac{\partial f_{1}}{\partial M_{1}} & =3 a_{1} n M_{1}^{2}-2 a_{1}(n+1) M_{1}+a_{1} V-\frac{a_{2} M_{2}}{\left(1+a_{3} M_{1}\right)^{2}}, \\
\frac{\partial f_{2}}{\partial M_{2}} & =b_{1}-\frac{2 b_{1} n M_{2}}{V}+\frac{b_{2} M_{1}}{1+a_{3} M_{1}}, \\
\left|\frac{\partial f_{1}}{\partial M_{1}}\right| & =\left|a_{1}\left(3 n M_{1}^{2}+V\right)-\left[2 a_{1}(n+1) M_{1}+\frac{a_{2} M_{2}}{\left(1+a_{3} M_{1}\right)^{2}}\right]\right|, \\
& <\left|a_{1}\left(3 n M_{1}^{2}+V\right)\right| \leq a_{1} V(3 n V+1) ; \\
\left|\frac{\partial f_{2}}{\partial M_{2}}\right| & =\left|b_{1}+\frac{b_{2} M_{1}}{1+a_{3} M_{1}}-\frac{2 b_{1} n M_{2}}{V}\right|<\left|b_{1}+\frac{b_{2} M_{1}}{1+a_{3} M_{1}}\right|<b_{1}+\frac{b_{2}}{a_{3}} .
\end{aligned}
$$

$\forall t \rightarrow \infty$, solutions of system (1) are unique according to the Lipschitz condition.

Furthermore, applying the Poincaré-Bendixson criterion, we have

$$
\begin{aligned}
\operatorname{div}\left(f_{1}, f_{2}\right)= & \frac{\partial f_{1}}{\partial M_{1}}+\frac{\partial f_{2}}{\partial M_{2}} \\
= & 3 a_{1} n M_{1}^{2}+a_{1} V+b_{1}+\frac{b_{2} M_{1}}{1+a_{3} M_{1}} \\
& -\left[2 a_{1}(n+1) M_{1}+\frac{2 b_{1} n M_{2}}{V}+\frac{a_{2} M_{2}}{\left(1+a_{3} M_{1}\right)^{2}}\right]
\end{aligned}
$$$$
\neq 0 \text {. }
$$

This indicates that system (1) has at least one periodic orbit lying in the phase plane $\left(M_{1}, M_{2}\right)$ for a given set of parameters value according to the Poincaré-Bendixson criterion.

\section{Model steady state}

In this section, we describe a steady state equilibrium of the system.

(i) The zero growth isoclines equations are given by

$$
\left\{\begin{aligned}
F= & a_{1} a_{3} n M_{1}^{3}+\left(a_{1} n-a_{1} a_{3} n V-a_{1} a_{3} V\right) M_{1}^{2}+\left(a_{1} a_{3} V^{2}-a_{1} n V-a_{1} V\right) M_{1} \\
& -a_{2} V M_{2}+a_{1} V^{2}=0 \\
G= & V\left(1-a_{3}\right) M_{1}-n M_{2}+a_{2} m M_{1} M_{2}+V=0
\end{aligned}\right.
$$

Based on (5), we know that interaction happens. The system may admit more than one positive solution in the first quadrant as portrayed in Fig. 1 and Fig. 2. The ideal scenario is to choose parameter value such that the intersection point of the two isoclines lies in the positive quadrant. 


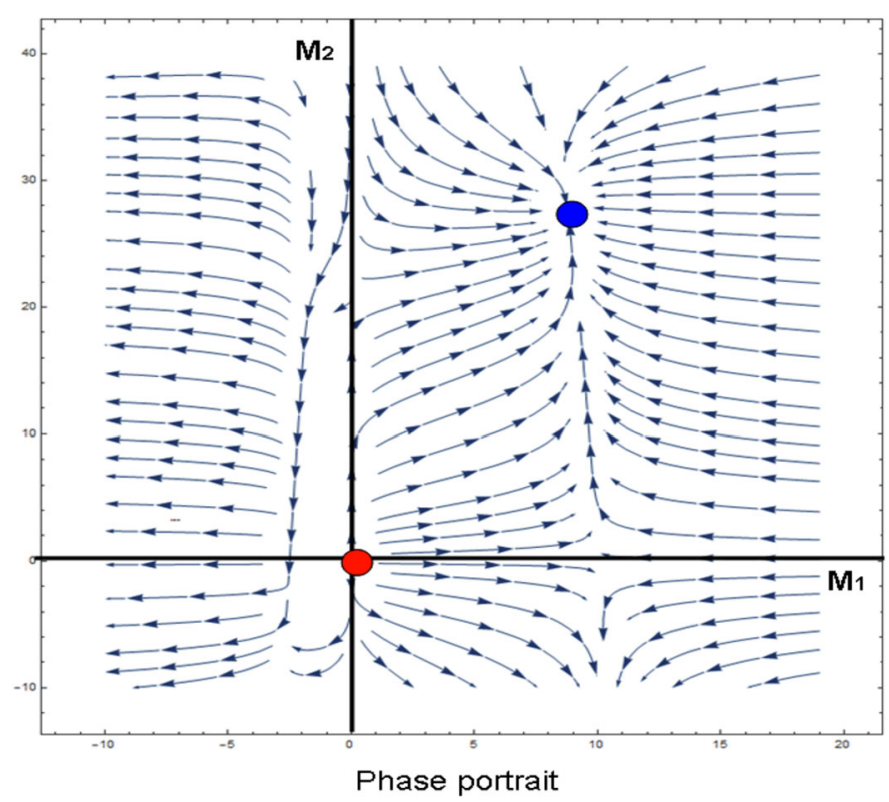

Figure 1 Phase portrait when the system is stable and admits a unique positive equilibrium point attracting all nearby trajectories

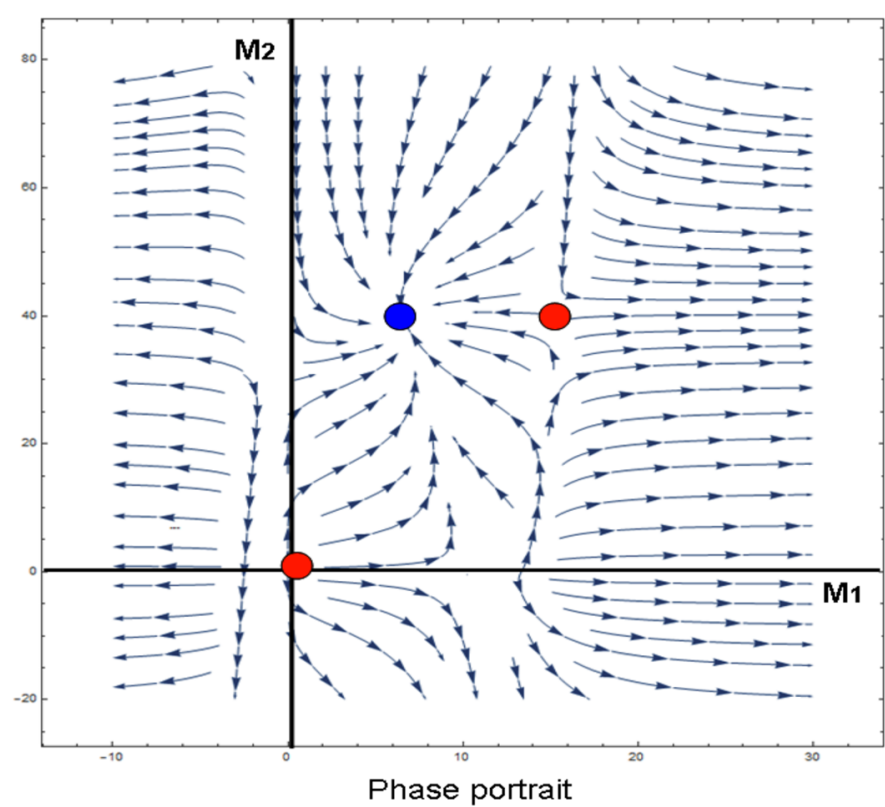

Figure 2 Phase portrait when the system admits one stable positive equilibrium point attracting nearby trajectories and one positive unstable equilibrium behaving as a saddle node

(ii) The unique positive equilibrium point of the system can be computed solving (5). From $G$ we obtain

$$
\begin{aligned}
& M_{2}^{*}=\frac{V\left(1+M_{1}^{*}+a_{3} M_{1}^{*}\right)}{n-a_{2} m M_{1}^{*}}, \quad n>a_{2} m M_{1}^{*}, \\
& t \rightarrow \infty, \quad M_{1}(t)<\frac{n}{a_{2} m} .
\end{aligned}
$$


After substituting in $F$ and rearranging, we get

$$
p_{0} M_{1}^{4}+p_{1} M_{1}^{3}+p_{2} M_{1}^{2}+p_{3} M_{1}+p_{4}=0
$$

where

$$
\begin{aligned}
& p_{0}=-a_{1} a_{2} n m, \\
& p_{1}=-a_{1}\left[a_{2} m n^{2}+a_{2} m\left(n-a_{3} n V-a_{3} V\right)\right], \\
& p_{2}=a_{1} n\left(n+n a_{3} V-a_{3} V\right)-a_{1} a_{2} m V\left(a_{3} V-n-1\right), \\
& p_{3}=V\left[a_{1} n\left(a_{3} V-n-1\right)-a_{2} V\left(a_{3}+1\right)-a_{1} a_{2} m V\right], \\
& p_{4}=\left(a_{1} n-a_{2}\right) V^{2} .
\end{aligned}
$$

Equation (7) could be reduced by changing variables to

$$
X^{4}+\mu_{0} X^{3}+\mu_{1} X+\mu_{2}=0
$$

where

$$
M_{1}=X-\frac{p_{1}}{4 p_{0}}
$$

and

$$
\begin{aligned}
& \mu_{0}=\frac{p_{2}}{p_{0}}-\frac{3 p_{1}^{2}}{8 p_{0}^{2}} \\
& \mu_{1}=\frac{p_{3}}{p_{0}}-\frac{p_{1} p_{2}}{2 p_{0}^{2}}+\frac{p_{1}^{3}}{8 p_{0}^{3}}, \\
& \mu_{3}=\frac{p_{4}}{p_{0}}-\frac{p_{1} p_{3}}{4 p_{0}^{2}}+\frac{p_{1}^{2} p_{3}}{16 p_{0}^{3}}-\frac{3 p_{1}^{4}}{256 p_{0}^{4}} .
\end{aligned}
$$

Take a variable $s$ satisfying the following resolving cubic equation:

$$
8 s^{3}-4 \mu_{0} s^{2}-8 \mu_{2} s+\left(3 \mu_{0} \mu_{2}-\mu_{1}^{2}\right)=0 .
$$

It follows

$$
\left(X^{2}+s\right)^{2}-\left[\left(2 s-\mu_{0}\right) X^{2}-\mu_{1} X+s^{2}-\mu_{2}\right]=0
$$

and

$$
\left(2 s-\mu_{0}\right) X^{2}-\mu_{1} X+s^{2}-\mu_{2}=\left(2 s-\mu_{0}\right)\left(X-X^{+}\right)\left(X-X^{-}\right) .
$$

Under the constraint $X^{+}=X^{-}$, we can write

$$
\left(X^{2}+s+\sqrt{2 s-\mu_{0}} X-\frac{\mu_{1}}{2 \sqrt{2 s-\mu_{0}}}\right)\left(X^{2}+s-\sqrt{2 s-\mu_{0}} X+\frac{\mu_{1}}{2 \sqrt{2 s-\mu_{0}}}\right)=0
$$


Equation (8) admits four solutions given by

$$
\begin{aligned}
& X_{1,2}=-\frac{1}{2} \sqrt{2 s-\mu_{0}} \pm \frac{1}{2} \sqrt{-2 s-\mu_{0}+\frac{2 \mu_{1}}{\sqrt{2 s-\mu_{0}}}} \\
& X_{3,4}=\frac{1}{2} \sqrt{2 s-\mu_{0}} \pm \frac{1}{2} \sqrt{-2 s-\mu_{0}-\frac{2 \mu_{1}}{\sqrt{2 s-\mu_{0}}}} .
\end{aligned}
$$

From (13) and (14) we deduct

$$
M_{1}^{k}=X_{k}-\frac{\mu_{1}}{4 \mu_{0}}, \quad k=1,2,3,4 .
$$

Then the steady state equilibrium is given by

$$
\left(M_{1}^{*}, M_{2}^{*}\right)=\left(M_{1}^{k}, \frac{V\left(1+M_{1}^{k}+a_{3} M_{1}^{k}\right)}{n-a_{2} m M_{1}^{k}}\right), \quad k=1,2,3,4 .
$$

\section{Model stability analysis}

In this section, we determine stability conditions around the system steady state equilibrium and analyze interaction outcome.

Let the Jacobian matrix be

$$
J=\left(\begin{array}{ll}
O & P \\
Q & R
\end{array}\right)
$$

where

$$
\begin{aligned}
& O=3 a_{1} n \bar{M}_{1}^{2}-2 a_{1}(n+1) \bar{M}_{1}+a_{1} V-\frac{a_{2} \bar{M}_{2}}{\left(1+a_{3} \bar{M}_{1}\right)^{2}} \\
& P=-\frac{a_{2} \bar{M}_{1}}{1+a_{3} \bar{M}_{1}}, \quad Q=\frac{b_{2} \bar{M}_{2}}{\left(1+a_{3} \bar{M}_{1}\right)^{2}} \\
& R=b_{1}-\frac{2 b_{1} \bar{M}_{2}}{V}+\frac{b_{2} \bar{M}_{1}}{1+a_{3} \bar{M}_{1}} .
\end{aligned}
$$

1 -At the origin, we have

$$
J(0,0)=\left(\begin{array}{cc}
a_{1} V & 0 \\
0 & b_{1}
\end{array}\right)
$$

The origin is always unstable, the polynomial equation admits two positive roots and (19) determinant is greater than zero.

2-At the positive equilibrium point, we have

$$
\begin{aligned}
& O=3 a_{1} n M_{1}^{* 2}+a_{1} V-\left[2 a_{1}(n+1) M_{1}^{*}+\frac{a_{2} M_{2}^{*}}{\left(1+a_{3} M_{1}^{*}\right)^{2}}\right] \\
& P=-\frac{a_{2} M_{1}^{*}}{1+a_{3} M_{1}^{*}}, \quad Q=\frac{b_{2} M_{2}^{*}}{\left(1+a_{3} M_{1}^{*}\right)^{2}} \\
& R=b_{1}+\frac{b_{2} M_{1}^{*}}{1+a_{3} M_{1}^{*}}-\frac{2 b_{1} M_{2}^{*}}{V} .
\end{aligned}
$$




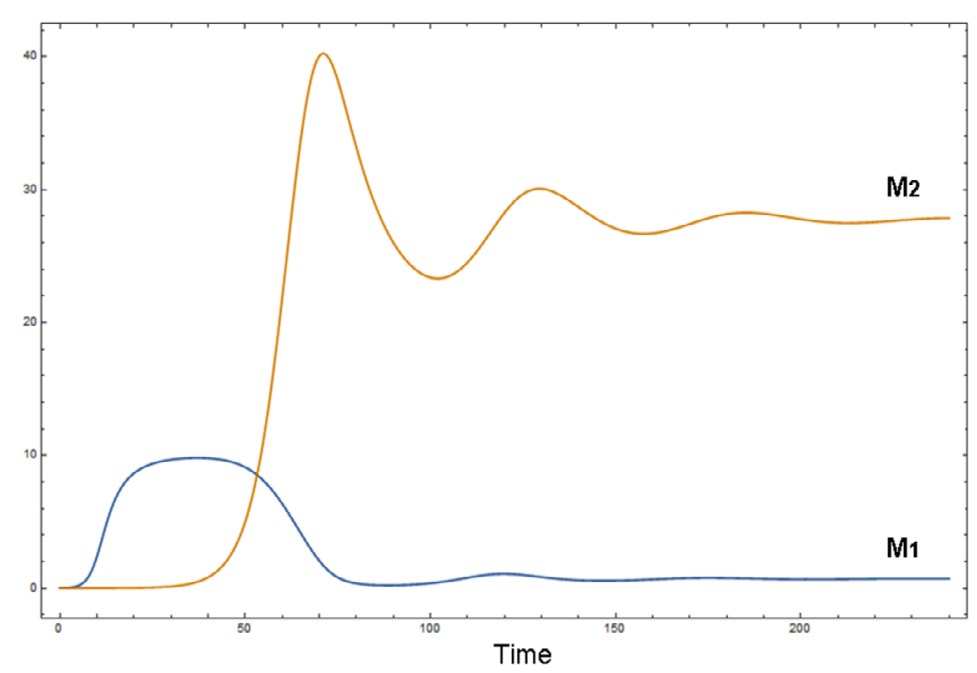

A

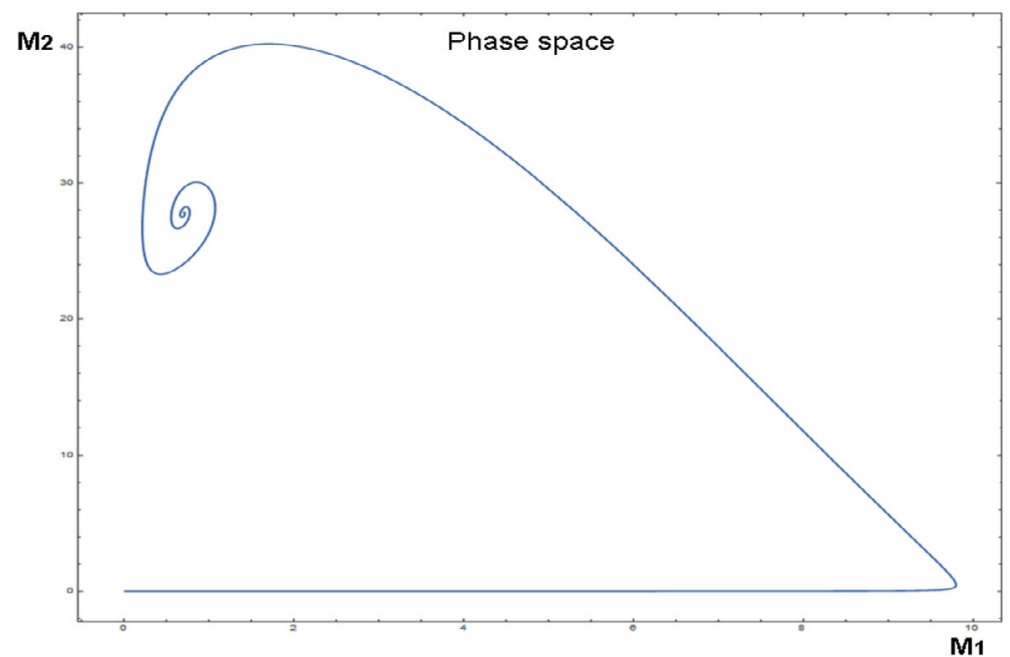

B

Figure 3 Time series showing local stability of the system at the steady state equilibrium neighborhood in $\mathrm{A}$ and the corresponding phase space in $B$ for $M_{1}(0)=0.01, M_{2}(0)=0.002, a_{1}=0.06, b_{1}=0.039, V=10$, $a_{2}=0.023, b_{2}=0.06, a_{3}=0.3, n=0.79, m=0.68$

The characteristic polynomial equation is given by

$$
\lambda^{2}-(O+R) \lambda+O R-P Q=0 .
$$

The system is asymptotically stable at the neighborhood of the steady state equilibrium only if $O+R<0$ and $O R-P Q>0$.

In case equation (21) admits complex conjugate imaginary roots of the form $\lambda=\mu \pm i \omega$, we have

$$
\left\{\begin{array}{l}
\mu^{2}-\omega^{2}-\mu(O+R)+O R-P Q=0 \\
2 \mu=O+R
\end{array}\right.
$$




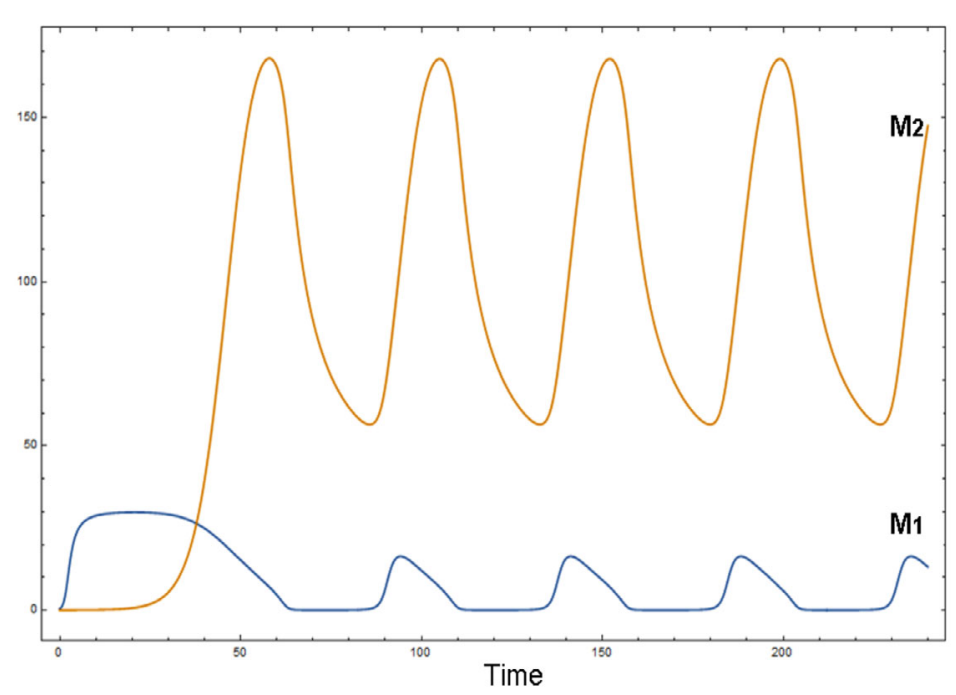

A

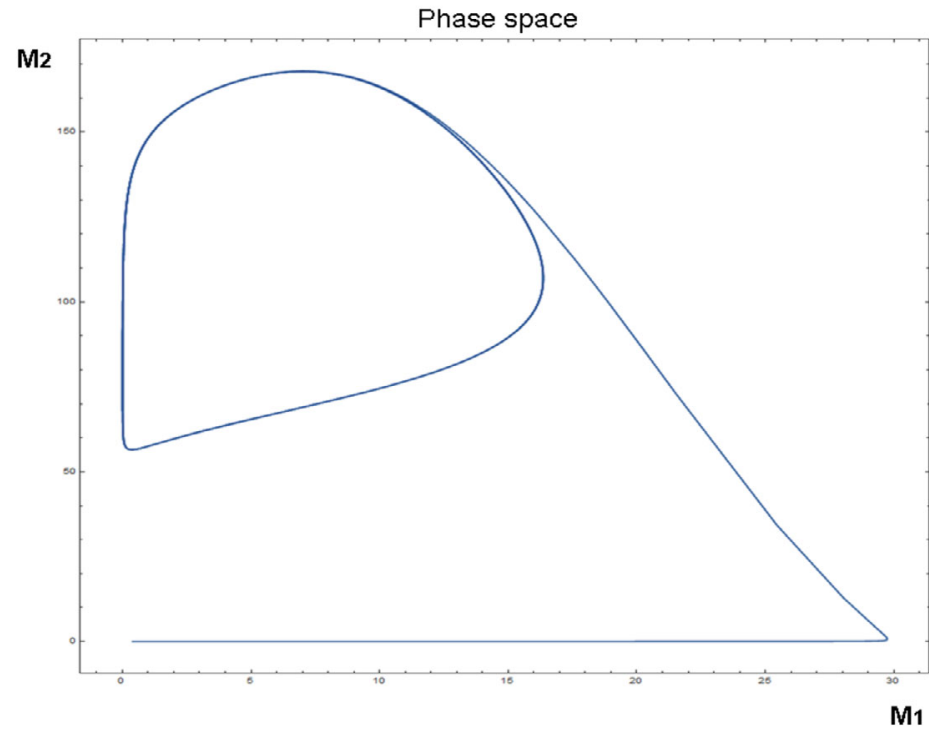

B

Figure 4 Time series showing the existence of periodic solutions at the steady state equilibrium neighborhood $A$ and the phase portrait in $B$ for $M_{1}(0)=0.39, M_{2}(0)=0.01, a_{1}=0.06, b_{1}=0.039, V=30$, $a_{2}=0.023, b_{2}=0.06, a_{3}=0.3, n=0.9, m=0.8$

$$
\left\{\begin{array}{l}
\mu=(O+R) / 2 \\
\omega= \pm \sqrt{(O+R)^{2} / 4-(O+R) / 2+O R-P Q}
\end{array}\right.
$$

If $\mu=0$, then a Hopf bifurcation occurs at $\left(M_{1}^{*}, M_{2}^{*}\right)$ and all solution curves lie on $\omega=$ $\pm \sqrt{O R-P Q}$ as far as $O R>P Q$. If $\mu \neq 0$, then at $\left(M_{1}^{*}, M_{2}^{*}\right)$ neighborhood solution curves are spiraling inward or outward depending on $\mu$ sign.

\section{Numerical results}

In this section, to test the proposed model predictability and validate the theoretical hypothesis, we carried out computer simulation (Figs. 3, 4, 5, 6, 7) and used data collected from the Chinese social network media Weibo (Figs. 8, 9, 10). Several situations of interac- 


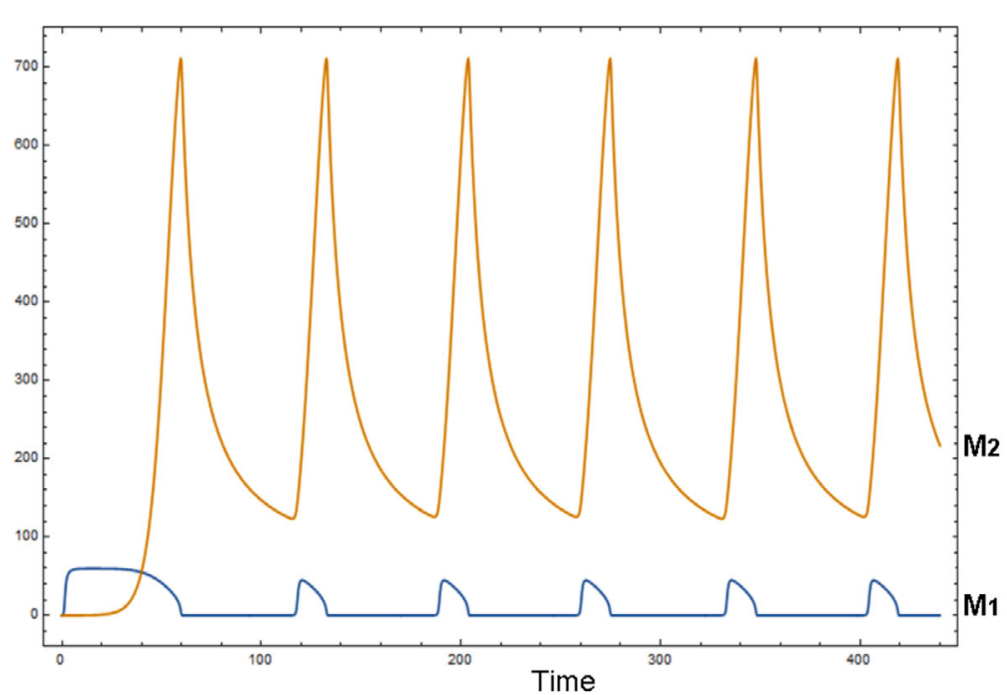

A

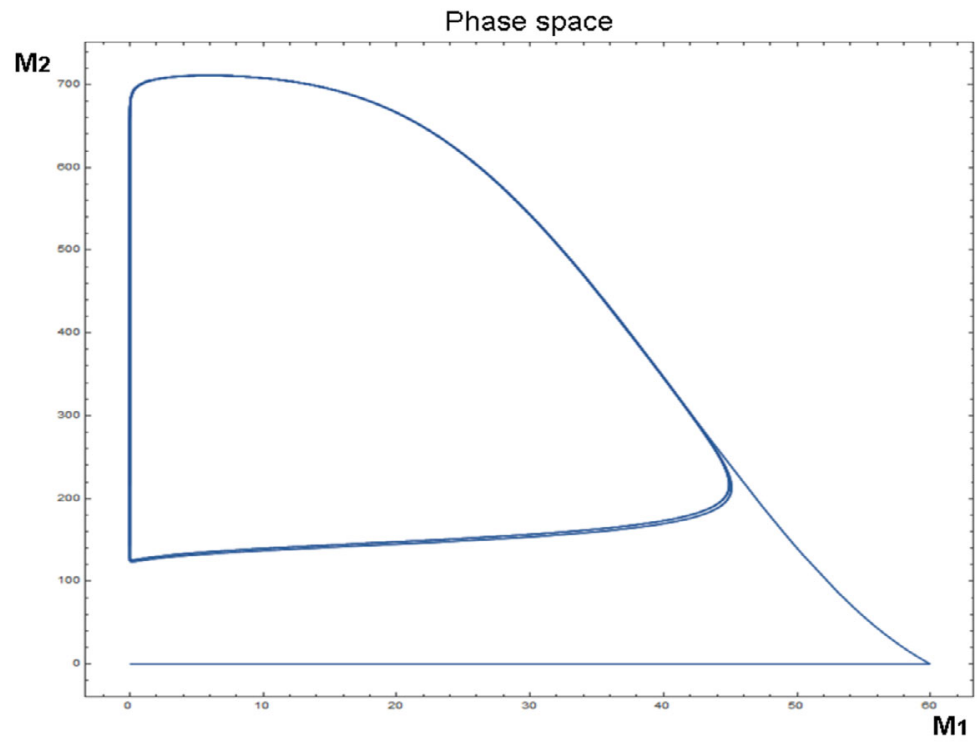

B

Figure 5 Time series showing the existence of periodic solutions at the steady state equilibrium neighborhood giving birth to a stable limit cycle in $A$ and the phase portrait in $B$ for $M_{1}(0)=0.09, M_{2}(0)=0.02$, $a_{1}=0.07, b_{1}=0.015, V=60, a_{2}=0.023, b_{2}=0.06, a_{3}=0.3, n=0.9, m=0.8$

tion were considered. In all the scenarios, model parameters are constant. We computed $M_{1}$ and $M_{2}$ intrinsic growth factors respectively $a_{1}=0.03, b_{1}=0.9$ as per capita increase of the number of views per unit time (here per day, after rescaling the data for practical purpose). Computing $a_{2}, b_{2}$, and $a_{3}$ is more challenging using the data at hand, $a_{2}$ being the effect $M_{2}$ has on $M_{1}$, modeling $M_{1}$ per capita decrease in the number of views. $b_{2}$ is the positive feedback resulting from this interaction, while $a_{3}$ captures relative speed of increase in the number of views per unit time for $M_{2}$. This implies that $M_{2}$ needs more time to benefit from positive feedback at high $M_{1}$ density. We varied initial conditions, interaction coefficients, system carrying capacity, and control parameters $m$ and $n$ to test system sensitivity to small perturbations and explore the interaction outcome. 


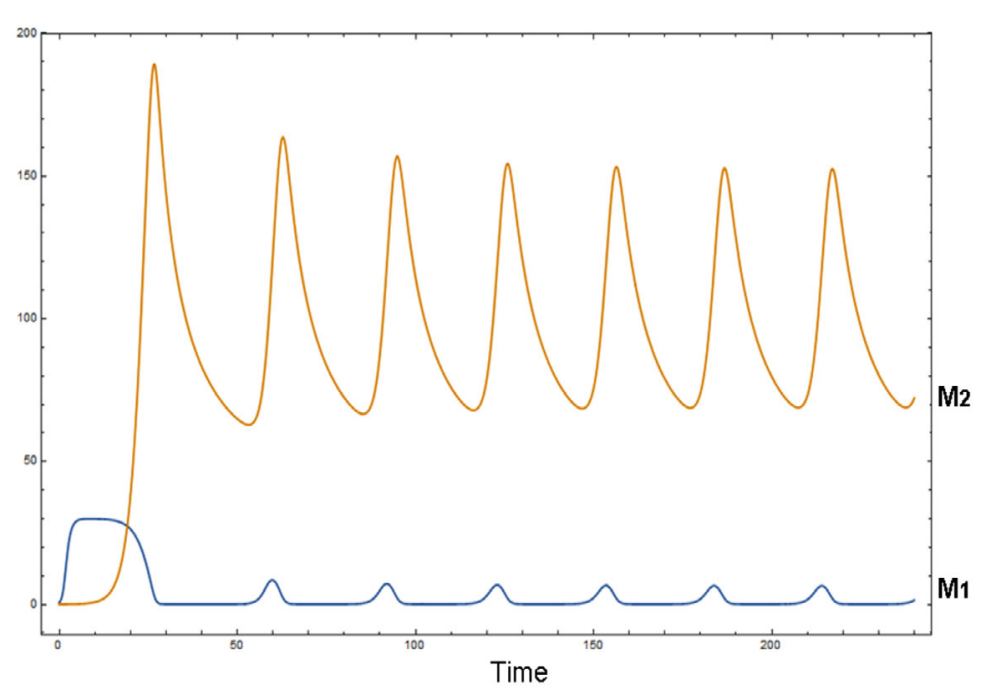

A

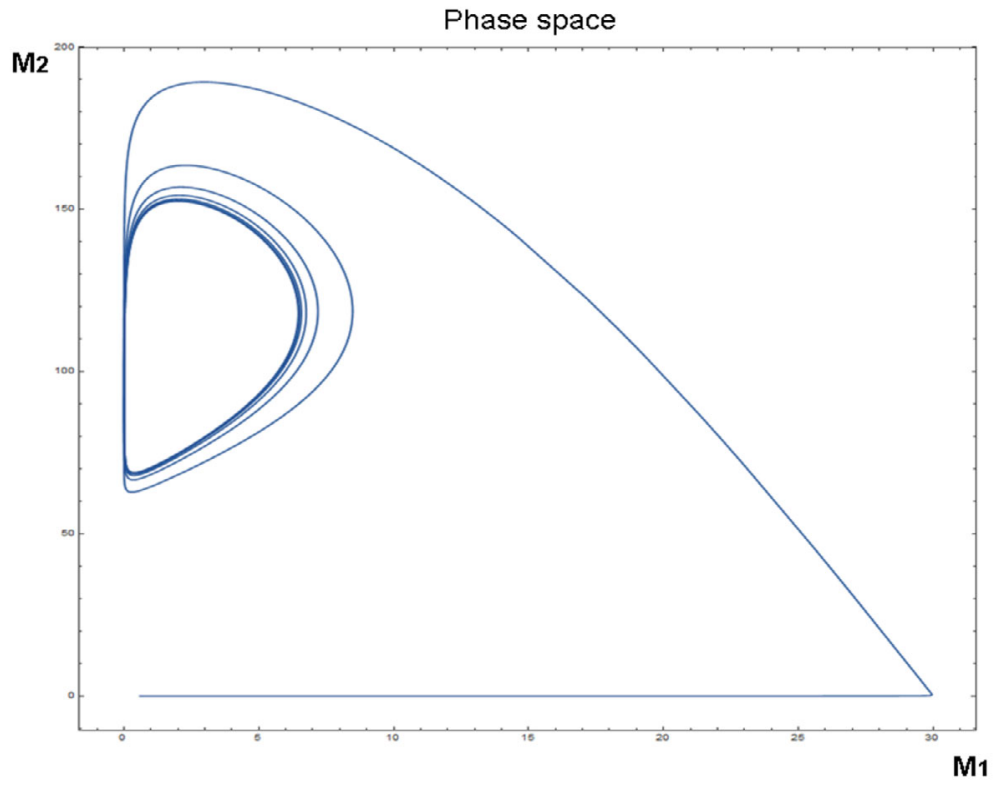

B

Figure 6 Time series showing the existence of periodic solutions at the vicinity of the steady state equilibrium giving birth to a stable limit cycle in $A$ and the phase portrait in $B$ for $M_{1}(0)=0.6, M_{2}(0)=0.02$, $a_{1}=0.07, b_{1}=0.039, V=30, a_{2}=0.023, b_{2}=0.06, a_{3}=0.13, n=0.49, m=0.68$

1-Fig. 3 displays the dynamic of interaction when system is stable and there is peaceful coexistence for a given set of parameters value. Benefiting from a larger audience and a more reliable publisher, namely the authority, $M_{2}$ gets more views and stays attractive for a longer period of time. This validates the fact that releasing authoritative information containing the truth at a specific time is important to reduce the negative effect of persistent rumor or fake news. Control parameters $m$ and $n$ have significant impact on system dynamic in respect to per capita growth rate or the number of views per unit time. Adjusting these control parameters by improving exposition and stimulating readers' interest using marketing tools and techniques may result in enhancing the attractiveness of respective messages and stabilizing the system. As portrayed in Fig. 4, when we vary $M_{2}$ harvesting 


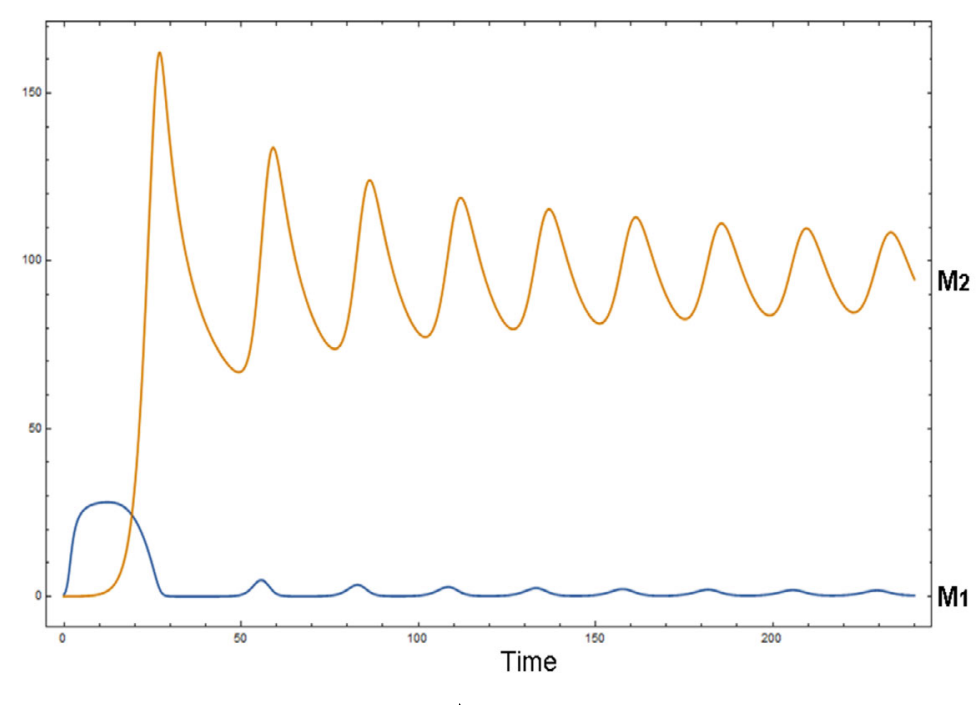

A

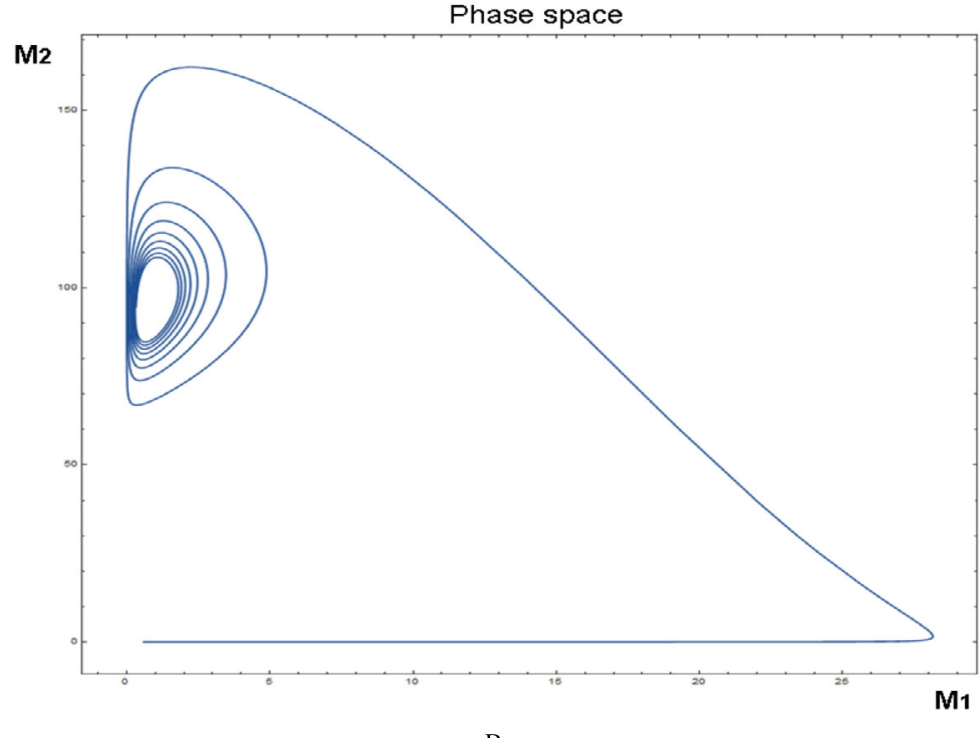

$\mathrm{B}$

Figure 7 Time series showing the existence of periodic solutions at the neighborhood of the steady state equilibrium giving birth to a stable limit cycle in $A$ and the phase portrait in $B$ for $M_{1}(0)=0.6, M_{2}(0)=0.02$, $a_{1}=0.07, b_{1}=0.039, V=30, a_{2}=0.023, b_{2}=0.06, a_{3}=0.13, n=0.99999, m=0.68$

coefficient significantly, the system exhibits rich dynamical behavior. The existence of periodic orbits and stable limit cycle at the vicinity of the unique positive equilibrium point, as illustrated in Figs. 5, 6, and 7, is interesting from system control perspective [25, 26].

As interacting messages have strong correlation due to resources limitation, varying $m$ and $n$ will result in affecting significantly the amplitude of oscillations and directly the limit cycle at the vicinity of the steady state equilibrium. Adjusting these parameters may end up in optimizing resources allocation in a closed environment when competition is intensive to maintain system stability, as portrayed in Figs. 6 and 7.

Readers' interest for a subject will drop when there are more choices as competition will intensify. To reach a larger number of views, choosing trusted and reliable publishers is a 
key in controlling information propagation among social networks targeted community or reducing propagating rumors negative effects on human mind and social wellbeing.

To support the decision in promoting broadcasted authoritative information and reduce the negative effect of rumor, decision makers need to analyze targeted community's social ties, network structure and to release the curative content at the right time. The key control parameters $m$ and $n$ will be difficult to determine scientifically with accuracy, as they are related to users' behavior which is stochastic in nature.

2 -By varying control parameters $m$ and $n$, interaction coefficient value and rescaling population size to 100, we obtained feasible results illustrated in Figs. 8, 9, and 10. A stable coexistence between the current rumor and the related authoritative information is shown. After the release of $M_{2}$ via the authority, the trending $M_{1}$ containing harmful contents shrinks at relative speed as more and more users enter in contact with $M_{2}$ as displayed in Fig. 8. By choosing $a_{3}=0.13, n=0.7, m=0.01$, we gave $M_{2}$ more time to assimilate captured resources and benefit from interaction, here attracting more users, as shown in Fig. 9. When the authoritative information is relatively attractive to the targeted community, the amplitude of oscillations is significantly affected.
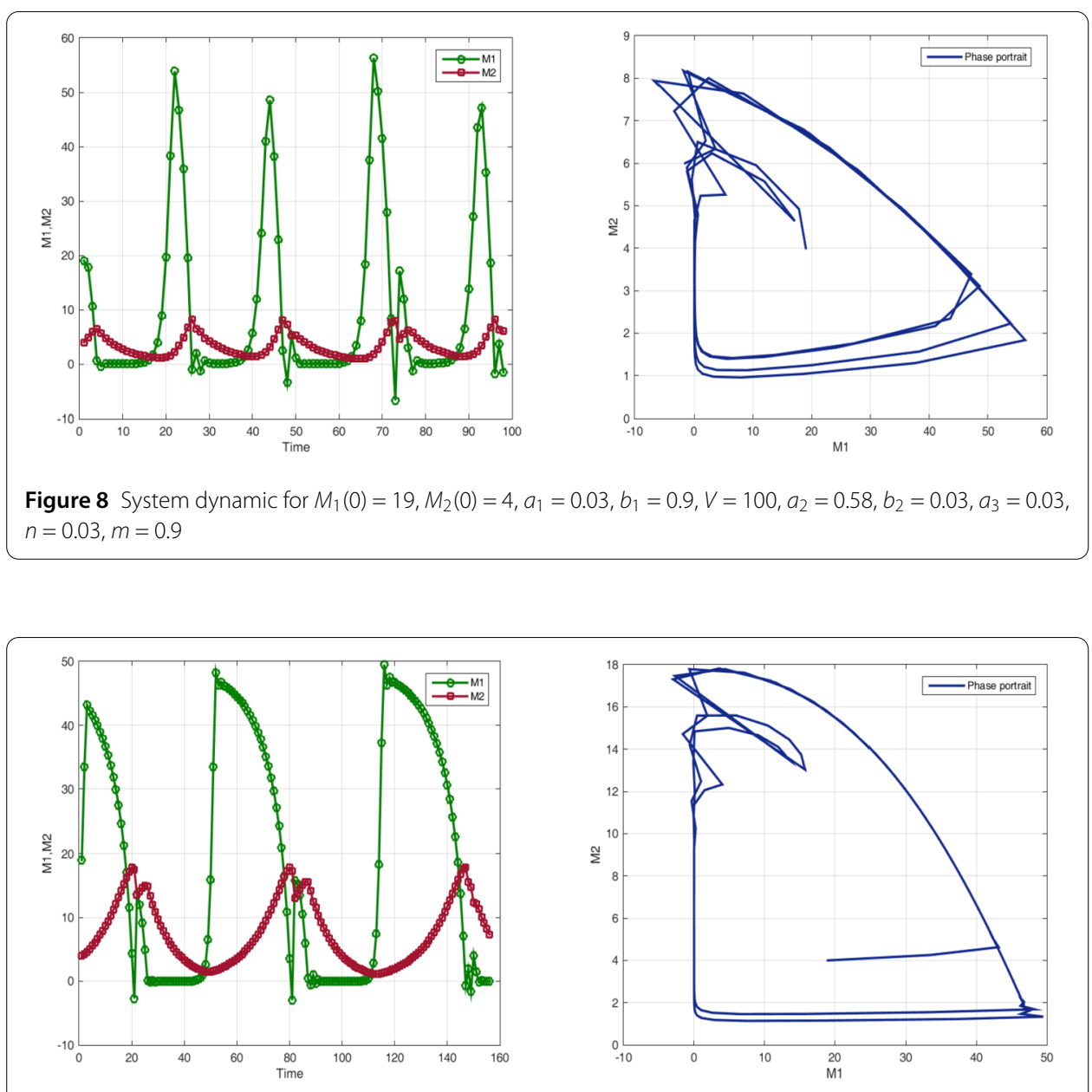

Figure 9 System dynamic for $M_{1}(0)=19, M_{2}(0)=4, a_{1}=0.03, b_{1}=0.9, V=100, a_{2}=0.3, b_{2}=0.03, a_{3}=0.13$, $n=0.7, m=0.01$ 

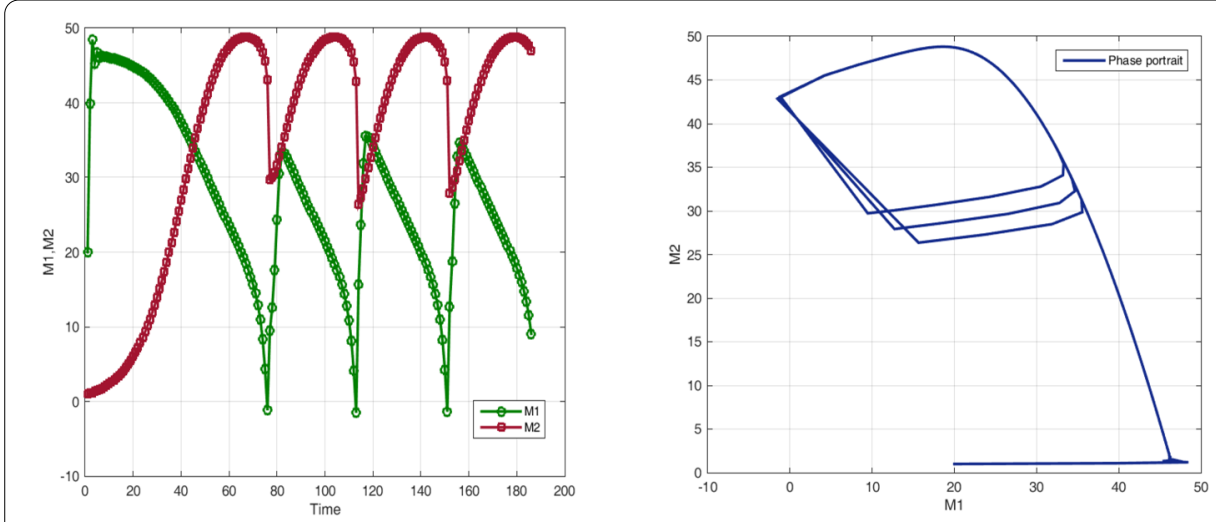

Figure 10 Dynamics of interaction for $M_{1}(0)=20, M_{2}(0)=1, a_{1}=0.03, b_{1}=0.9, V=100, a_{2}=0.16, b_{2}=0.07$, $a_{3}=0.32, n=0.99, m=0.2$

Figure 10 illustrates an ideal scenario for $a_{2}=0.16, b_{2}=0.07, a_{3}=0.32, n=0.99, m=0.2$, where the authoritative information overpowered the persistent rumor $M_{1}$, resulting in less and less people propagating the latter. However, even when the majority of people are reading and propagating $M_{2}$, there are still users who continue to be interested in $M_{1}$, which is consistent with human mind and people inclination to experiment themselves what they hear about.

\section{Conclusion}

In this paper, to model the interaction effect of two messages traveling through social networks sharing the same interest for readers, we proposed a two-species logistic interactive model with Holling type functional response and maturation parameters for both interacting species. We considered respective attractiveness and thresholds for each message in respect to logistic growth and control parameters. We showed that controlling a strategic node or a publisher with highest reliability has a direct influence on system stability and resources availability for covering a larger audience in the targeted community. By applying stability theory and studying the system qualitatively, we found in numerical results that the system exhibited rich dynamical behavior, interaction dynamics was predictable, and the system could be stable for a given set of parameters value. Both species' dynamics are submitted to initial conditions, resources availability, marketing strategy, and publisher reliability through respective control parameters. From the field data at hand, we showed that maturation parameters and assimilation coefficient have significant impact on the steady state equilibrium dynamics, as the amplitude of oscillations changes when we vary respective parameters, shaping the system's asymptotic and global behavior.

Acknowledgements

This work is supported by National Social Science Foundation of China (No. 18BXW118).

Funding

We declare that no founder has influenced the design, the analysis, and the interpretation of data of the work presented in this article.

Availability of data and materials

Data and codes generated and used in this study are available from the corresponding author upon request. 
Authors' contributions

YZ, FL, and YHK designed experiments; WH, YZ, and YHK carried out experiments and validated experimental results. FL and WH prepared Figs. 1-10; YHK and FL interpreted experimental results; YHK wrote the manuscript. All authors read and approved the final manuscript.

\section{Author details}

${ }^{1}$ School of Economics and Management, Yanshan University, Qinhuangdao, China. ${ }^{2}$ School of Public Administration, Hengshui University, Hengshui, China. ${ }^{3}$ Center for Internet Plus and Industry Development, Yanshan University, Qinhuangdao, China.

\section{Publisher's Note}

Springer Nature remains neutral with regard to jurisdictional claims in published maps and institutional affiliations.

\section{Received: 5 February 2020 Accepted: 15 June 2020 Published online: 29 June 2020}

\section{References}

1. Morgan \& Claypool synthesis lectures on data management, Oct. 2013

2. Morstatter, F., Kumar, S., Liu, H., Maciejewski, R.: Understanding Twitter data with TweetXplorer. In: Proceedings of the 19th ACM SIGKDD International Conference on Knowledge Discovery and Data Mining, KDD'13, pp. 1482-1485. ACM, New York (2013)

3. Netten, N., van Someren, M.: Identifying segments for routing emergency response dialogues. In: 5th International Conference on Information Systems for Crisis Response and Management (ISCRAM) (2008)

4. Wang, F., Wang, H., Xu, K.: Diffusive logistic model towards predicting information diffusion in online social networks (2001). arXiv:1108.0442

5. Yang, J., Counts, S.: Predicting the speed, scale, and range of information diffusion in Twitter. In: Proceedings of the Fourth International Conference on Weblogs and Social Media (2010)

6. Jiang, J., Wilson, C., Wang, X., Huang, P., Sha, W., Dai, Y., Zhao, B.Y.: Understanding latent interactions in online socia networks. In: Proceedings of the 10th Annual Conference on Internet Measurement, IMC '10, pp. 369-382. ACM, New York (2010)

7. Myers, S.A., Zhu, C., Leskovec, J.: Information diffusion and external influence in networks (2012). arXiv:1206.1331

8. Anagnostopoulos, A., Brova, G., Terzi, E.: Peer and authority pressure in information-propagation models (2011)

9. Tang, S., Yuan, J., Mao, X., Li, X.-Y., Chen, W., Dai, G.: Relationship classification in large scale online social networks and its impact on information propagation. In: INFOCOM, pp. 2291-2299 (2011)

10. Bordia, P., DiFonzo, N.: Problem solving in social interactions on the Internet: rumor as social cognition. Soc. Psychol. Q. 67, 33-49 (2004)

11. Dyagilev, K., Mannor, S., Elad, Y.-T.: Generative models for rapid information propagation. In: 1st Workshop on Social Media Analytics, pp. 35-43. ACM, New York (2010)

12. Moreno, Y., Nekovee, M., Pacheco, A.F.: Dynamics of rumor spreading in complex networks. Phys. Rev. E 69, Article ID 066130 (2004)

13. Xiang, B., Liu, Q., Chen, E., Xiong, H., Zheng, Y., Yang, Y.: Page rank with priors: an influence propagation perspective. In: IJCAI (2013)

14. Liu, Q., Xiang, B., Chen, E., Xiong, H., Tang, F., Yu, J.X.: Influence maximization over large-scale social networks: a bounded linear approach. In: CIKM'14, pp. 171-180. ACM, New York (2014)

15. Wang, H., Wang, P., Tamer Şenel, M., Li, T.: On novel nonhomogeneous multivariable grey forecasting model NHMGM Math. Probl. Eng. 2019, Article ID 9049815 (2019)

16. Chaoji, V., Ranu, S., Rastogi, R., Bhatt, R.: Recommendations to boost content spread in social networks. In: Proceedings of the 21st International Conference on World Wide Web, pp. 529-538. ACM, New York (2012)

17. Wei, D., Zhou, T., Cimini, G., Wu, P., Liu, W., et al.: Effective mechanism for social recommendation of news. Physica A $390,2117-2126(2011)$

18. Chen, D.B., Gao, H.: An improved adaptive model for information recommending and spreading. Chin. Phys. Lett. 29 Article ID 048901 (2012)

19. Goncalves, B., Perra, N., Vespignani, A.: Modeling users activity on Twitter networks: validation of Dunbar's number. PLOS ONE 6, Article ID e22656 (2011)

20. Weng, L., Ratkiewicz, J., Perra, N., Goncolves, B., Castillo, C., et al.: The role of information diffusion in the evolution of social network. In: Proceedings of the 19th ACM SIGKDD International Conference on Knowledge Discovery and Data Mining, KDD'13, pp. 356-364. ACM, New York (2013)

21. Miller, D.A., Grand, J.B., Fondell, T.F., Anthony, M.: Predator-prey functional response and prey survival: direct and indirect interactions affecting a marked prey population. J. Anim. Ecol. 75, 101-110 (2006)

22. Xiao, D., Zhang, Z.: On the uniqueness and nonexistence of limit cycles for predator-prey systems. Nonlinearity 16, $1185-1201$ (2003)

23. Krivan, V., Eisner, J.: The effect of Holling type II functional response on apparent competition. Theor. Popul. Biol. 70 421-430 (2006)

24. Zhang, Y., Koura, Y.H., Su, Y.: Dynamic of a delayed predator-prey model with application to network' users' data forwarding. Sci. Rep. 9, Article ID 12535 (2019)

25. Li, T., Rogovchenko, Y.V. On the asymptotic behavior of solutions to a class of third-order nonlinear neutral differential equations. Appl. Math. Lett. 105, Article ID 106293 (2020)

26. Chiu, K.-S., Li, T.: Oscillatory and periodic solutions of differential equations with piecewise constant generalized mixed arguments. Math. Nachr. 292, 2153-2164 (2019) 